\title{
Pharmacogenetics: global clinical markers
}

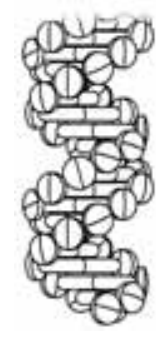

\author{
Sharan Marsh \\ Washington U niversity School \\ of M edicine, Campus Box \\ 8069,660 S. Euclid Ave, \\ St. Louis, M 0 63110, USA \\ Tel.: +1 314747 5186; \\ Fax: +1 314362 3764; \\ E-mail: smarsh@ \\ im.wustl.edu
}

\author{
'Allele frequencies for most \\ polymorphisms show signific ant \\ differences between populations, \\ and within common population \\ classific ations.'
}

The release of the first working draft of the human genome was quickly followed by the prediction that approximately 1.42 million SN Ps would be identified in the human genome [1]. This estimate was rapidly exceeded. Currently, the $\mathrm{N}$ ational $\mathrm{C}$ enter for Biotechnology Information (NCBI) polymorphism repository dbSN $\mathrm{P}$, has well over 4 million polymorphisms [101]. Endeavours, such as the International $\mathrm{H}$ apM ap project [102], have provided insights into not only the quantity of polymorphisms, but also the variability in polymorphism frequency betwen world populations (specifically Europe, Yoruba, Japan and China) [2]. The available data set for pharmacogenetics research will be further expanded with the 1000 Genomes Project [103], which aims to sequence the entire genome of 1000 individuals from multiple populations (chiefly the extended $\mathrm{H}$ apM ap sample set). This will provide a highly detailed map of polymorphisms that occur at a frequency of $1 \%$ or more in the human genome.

The ultimate goal of pharmacogenetics is to define robust markers that can be used in the clinical setting to aid in individualized treatment selection. However, despite the plethora of genetic information at our fingertips the true number of established pharmacogenetic markers ready for integration into clinical practice is remarkably small [3,4], and their usefulness may be geographically limited.

The lack of robust clinical pharmacogenetic markers could be owing to a multitude of reasons, including environmental factors, diet, concurrent medications, sample size, treatment regimen, population variation in allele frequencies and the lack of available sample sets to perform validation analyses.

In addition to the reasons above, commonly published polymorphisms often suffer from contradictory results, making their role in the clinic unclear. Sometimes this can be clarified by taking a closer look at the studies. For example, conflicting data for U GT $1 A 1 * 28$, an FDA approved marker for predicting toxicity in patients receiving the chemotherapy drug irinotecan, can be clarified by including irinotecan dose in the analysis [5]. The polymorphism appears to be a marker for neutropenia in patients receiving high-dose irinotecan, but is a less useful marker for patients receiving low-dose irinotecan.

Another factor involved in the use of U GT $1 A 1 * 28$ as a clinical marker is the allele fre quency difference between populations. Although UGT1A1*28 is a functional polymorphism and has been associated with irinote can toxicity in multiple populations [6], the allele frequency is lower in Asian populations (approximately $15 \%$ compared with $30-45 \%$ in Caucasian and African populations) [7]. Other potentially functional variants in UGTIA1 (e.g., U G T 1A 1*6) are prevalent in Asian populations and also demonstrate an association with irinotecan toxicity [8]. So although U GT 1A $1 * 28$ appears to be a relevant clinical marker for all populations, some information would be missed by screening this alone in every patient. There is still work needed to define a panel of markers for irinotecan toxicity in all populations.

'Frequenc ies of most described func tional polymorphisms have not been assessed in multiple populations, and

even where some comparison of populations has been performed, it is far from comprehensive.'

The differences in UGT1A1*28 allele frequency highlight a problem with many pharmacogenetic markers. Allele frequencies for most polymorphisms show significant differences between populations [9], and within common population classifications. Significant differences are found for GSTT $1 * 0$ allele frequencies (13-28\%) between countries with populations defined as Caucasian [10]. Evidence exists that allele frequencies between African populations can also differ significantly. The Gambia has one of the highest recorded allele 
frequencies for GSTP1 I105V (53\%) [11], whereas South Africa has one of the lowest (14\%) [12].

Even within countries differences can occur. For example Brazil, which has a highly admixed population, has significant frequency variability for polymorphisms in $\mathrm{N}$-acetyltransferase 2 (N AT 2) [13] and ABCB1 [14]. Although studies in the USA often stratify the data for European American and African-American patients, this is not frequently performed for analysis in other countries. This could lead to inadequate generalizations regarding allele frequencies in any given country or population classification.

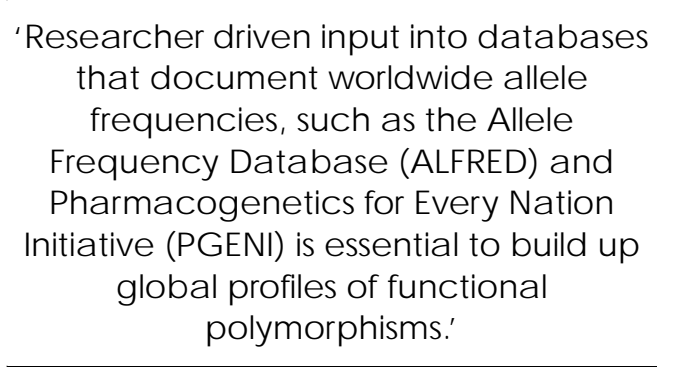

Another problem resulting from allele frequency variability between populations is the degree of linkage disequlibrium, and consequently haplotype structure can also vary significantly $[2,15]$. A tagSN $P$ in one population may not be a tagSN P for the same functional variant in a different population. Hence, clinically relevant polymorphisms need to be functional variants, not markers in linkage with a functional variant, as the linkage may breakdown between populations.

The most studied functional polymorphisms in a gene may not be appropriate to screen in every population or ethnic group, or may need to be subsidized with additional polymorphisms within the same gene. Polymorphisms in the genes encoding the enzyme thiopurine methyltransferase (TPM T) can be used to predict severe hematological toxicity from azathioprine drugs. H owever, the most commonly screened polymorphisms do not include TPM T*8, which contributes a significant proportion of functional T PM T alleles in Mozambique and Cabinda [16]. The functional CYP2D 6*4 allele has been associated with tamoxifen treatment outcome in breast cancer patients [17] and is prevalent in Caucasian populations (up to 20\%) [18]. An FD A advisory committee has recommended altering the tamoxifen package insert to include CYP2D 6 pharmacogenetic information [19]. H owever, it is still unclear which alleles are the most appropriate to test. CYP2D 6*4 is present at a lower fre quency, and even absent from many nonEuropean populations, where other nonfunction alleles, such as CYP2D 6*5, are more prevalent $[18,20]$. O ne study has assessed the nonfunctional alleles CYP2D $6 * 3, * 4, * 5$ or $* 6$ alleles and determined that patients heterozygous for these all eles have intermediate tamoxifen metabolism levels [21], and patients carrying CYP2D 6*4, $* 5, * 10$ or $* 41$ alleles have limited benefit from tamoxifen treatment [22]. A comprehensive study of the effects of CYP2D 6 nonfunctional and reduced function alleles on outcometo tamoxifen therapy including but not limited to CYP2D 6*4 needs to be performed to determine the full effect of CYP2D 6 polymorphisms.

Unfortunately, frequencies of most described functional polymorphisms have not been assessed in multiple populations, and even where some comparison of populations has been performed, it is far from comprehensive [23]. Future studies need to expand to assess multiple populations, and researcher driven input into databases that document worldwide allele frequencies, such as the Allele Frequency Database (ALFRED) [104] and Pharmacogenetics for Every $N$ ation Initiative (PGENI) [105] is essential to build up global profiles of functional polymorphisms. Panels of polymorphisms for each gene can then be constructed that can be screened in any population to identify patients at risk for toxicity and/or lack of efficacy prior to treatment selection.

Financial and competing interests disclosure The author is supported by the Pharmacogenetics Research N etwork U 01 GM 63340. The author has no other revant affiliations or financial involvement with any organization or entity with a financial interest in or financial conflict with the subject matter or materials discussed in the manuscript apart from those disclosed.

$\mathrm{N}$ o writing assistance was utilized in the production of this manuscript.

\section{Bibliography}

1. Sachidanandam $R$, Weissman $D$, Schmidt SC et al.: A map of human genome sequence variation containing 1.42 million single nucleotide polymorphisms. N ature 409, 928-933 (2001).
2. Frazer KA, Ballinger DG, Cox DR et al.: A second generation human haplotype map of over 3.1 million SN Ps. N ature 449, 851-861 (2007).

3. M arsh S, M CLeod H L: Pharmacogenomics: from bedside to clinical practice. $\mathrm{H}$ um. $\mathrm{M} \mathrm{Ol}$. Genet. 15, R89-R93 (2006).
4. Evans WE, M cLeod H L: Pharmacogenomics - drug disposition, drug targets, and side effects. N . Engl. J. M ed. 348, 538-549 (2003).

5. H oskins JM, G oldberg RM, Q u P, Ibrahim JG, M CLeod H L: U GT 1A1*28 genotype and irinotecan-induced 
neutropenia: dose matters. J. N atl Cancer Inst. 99, 1290-1295 (2007).

6. Innocenti F, Ratain M J: I rinotecan treatment in cancer patients with U GT $1 A 1$ polymorphisms. Oncology 17, 52-55 (2003).

7. H all D, Ybazeta G, D estro-Bisol G, Petzl-Erler M L, Di Rienzo A: Variability at the uridine diphosphate glucuronosyltransferase $1 \mathrm{~A} 1$ promoter in human populations and primates. Pharmacogenetics 9, 591-599 (1999).

8. H an JY, Lim H S, Shin ES et al.: Comprehensive analysis of U G T IA polymorphisms predictive for pharmacokinetics and treatment outcome in patients with non-small-cell lung cancer treated with irinotecan and cisplatin. J. Clin. O ncol. 24, 2237-2244 (2006).

9. Engen RM, M arsh S, van Booven DJ, $M$ CLeod $H L$ : Ethnic differences in pharmacogenetically relevant genes. Curr. D rug Targets 7, 1641-1648 (2006).

10. Garte S, Gaspari L, Alexandrie AK et al.: $M$ etabolic gene polymorphism frequencies in control populations. Cancer Epidemiol. Biomarkers Prev. 10, 1239-1248 (2001).

11. Wild CP, Yin F, Turner $P C$ et al.: Environmental and genetic determinants of aflatoxin-al bumin adducts in the $\mathrm{G}$ ambia. Int. J. Cancer 86, 1-7 (2000).

12. D andara $C$, Sayi J, M asimirembwa CM et al.: $G$ enetic polymorphism of cytochrome P450 1A1 (Cyp1A1) and glutathione transferases (M 1, T1 and $\mathrm{P} 1$ ) among
Africans. Clin. Chem. Lab. M ed. 40, 952-957 (2002).

13. Teixeira RL, M iranda $A B$, Pacheco $A G$ et al.: $G$ enetic profile of the arylamine $\mathrm{N}$-acetyltransferase 2 coding gene among individuals from two different regions of Brazil. M utat. Res. 624, 31-40 (2007).

14. Estrela RC, Ribeiro FS, Carvalho RS et al.: $D$ istribution of $A B C B 1$ polymorphisms among Brazilians: impact of population admixture. Pharmacogenomics 9, 267-276 (2008).

15. Jakobsson M, Scholz SW, Scheet P et al.: G enotype, haplotype and copy-number variation in worldwide human populations. N ature 451, 998-1003 (2008).

16. Oliveira E, Q uental $S$, Alves $S$, Amorim A, Prata M J: D o the distribution patterns of polymorphisms at the thiopurine S-methyltransferase locus in sub-Saharan populations need revision? $\mathrm{H}$ ints from Cabinda and M ozambique. Eur. J. Clin. Pharmacol. 63, 703-706 (2007).

17. Goetz M P, RaeJM, Suman VJ et al.: Pharmacogenetics of tamoxifen biotransformation is associated with clinical outcomes of efficacy and hot flashes. J. Clin. Oncol. 23, 9312-9318 (2005).

18. Gaedigk A, C oetsee C: The CYP2D 6 gene locus in South African Coloureds: unique allele distributions, novel alleles and gene arrangements. Eur. J. Clin. Pharmacol. (2008).

19. Reynolds S: SPORE study provides new guidelines for tamoxifen use. $\mathrm{N} \mathrm{Cl}$ Cancer Bulletin 3, 1-2 (2006).
20. Sistonen J, Sajantila A, Lao O, C orander J, Barbujani G, Fuselli S: CYP2D 6 worldwide genetic variation shows high frequency of altered activity variants and no continental structure. Pharmacogenet. Genomics 17 , 93-101 (2007).

21. Jin Y, D esta Z, Stearns V et al.: CYP2D 6 genotype, antidepressant use, and tamoxifen metabolism during adjuvant breast cancer treatment. J. N atl C ancer Inst. 97, 30-39 (2005).

22. Schroth W, Antoniadou L, Fritz P et al.: Breast cancer treatment outcome with adjuvant tamoxifen relative to patient CYP2D 6 and CYP2C 19 genotypes. J. Clin. Oncol. 25, 5187-5193 (2007).

23. $M$ arsh $S$, van Booven DJ, M CLeod H L: G lobal pharmacogenetics: giving the genome to the masses. Pharmacogenomics 7 , 625-631 (2006).

\section{Websites}

101. dbSN P summary page www.ncbi.nlm.nih.gov/SN P/snp_ summary.cgi

102. International $\mathrm{H}$ apM ap Project http://hapmap.org/

103. $1000 \mathrm{G}$ enomes Project www.1000genomes.org/index.html

104. Allele Frequency D atabase (ALFRED) http://alfred.med.yale.edu/alfred/index.asp

105. Pharmacogenetics for Every $\mathrm{N}$ ation Initiative (PGENI) www.pgeni.org 